\section{Losing symmetry by design}

\section{Kenneth D. M. Harris and Mark D. Hollingsworth}

CRYSTALline organic solids are becoming increasingly important for their optical, electrical and magnetic properties. In general, these depend not only on the properties of the constituent molecules, but also, sometimes critically, on the spatial arrangement of the molecules in the crystal. Progress in the design of molecular crystals with carefully tailored solid-state properties clearly relies upon an improved understanding of the factors that control crystal packing. Many successful, albeit largely empirical, strategies have been devised for engineering molecules to crystallize in controlled and predictable arrangements ${ }^{1}$. New advances in the 'crystal engineering' of hydrogenbonded structures, discussed by M. C. Etter (University of Minnesota) at a recent conference*, are now enabling the design of materials with important nonlinear optical properties.

Hydrogen bonds are ubiquitous in organic crystals and, because they are often stronger than other intermolecular forces, they frequently control the molecular packing arrangement. By surveying the extensive crystallographic data in the Cambridge Structural Database, Etter and co-workers have developed a systematic rationalization of hydrogen bonding patterns. They used graph theory to classify recurring patterns in a large number of hydrogen-bonded structures, allowing them to establish a set of empirical rules to categorize the features of hydrogen-bonded linkages formed by various specific types of functional groups. As a result, it is now possible to predict, with a good chance of success, the probable mode of crystal packing of molecules designed to have strategically arranged hydrogen-bond donor and acceptor groups.

A principal goal for Etter's group is the design of frequency-doubling crystals. Because these crystals convert light of one frequency to light with twice the frequency, they can greatly increase the frequency range of lasers, and have numerous potential applications in the emerging field of optoelectronics. The parameter that characterizes the usefulness of an individual molecule for frequency doubling - otherwise known as second-harmonic generation - is its second-order hyperpolarizability tensor, denoted $\beta$.

Molecular features such as polarizable $\pi$-electrons, donor-acceptor conjugate pairs or long conjugated chains generally give rise to a large value of $\beta$. In a crystalline material, however, second-harmonic

* 9th International Conference on the Chemistry of the Organic Solid State, Como, Italy, 2-7 July 1989. generation also depends on the spatial arrangement of the molecules. For example, a crystal cannot exhibit secondharmonic generation if its structure has a centre of symmetry, even if the constituent molecules have a large $\beta$. Further crystallographic criteria have been identified by Zyss and Oudar', and other factors, such as phase-matchability and

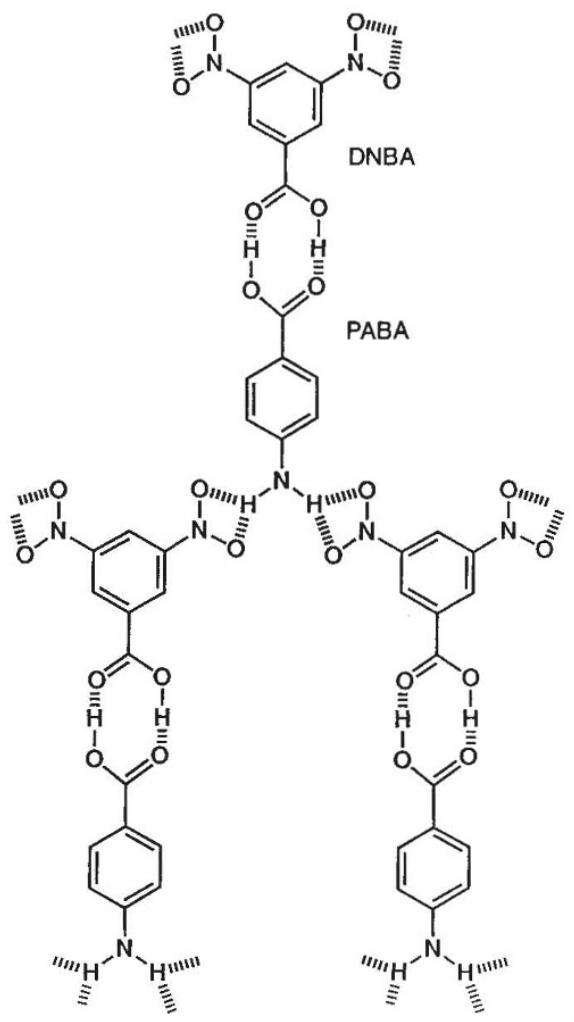

The co-crystal of $p$-aminobenzoic acid (PABA) and 3,5-dinitrobenzoic acid (DNBA) comprises a stacking of layers of the type shown. Each layer contains an extensively hydrogenbonded, non-centrosymmetric arrangement of the molecules. (The hydrogen bonds are indicated by dashed lines.)

absorptivity, also determine the ultimate usefulness of a material for frequency doubling.

Unfortunately, although in some ways predictably, many molecules that have a large value of $\beta$ tend to crystallize spontaneously in centrosymmetric structures: hence the interest of Etter and others in developing crystal engineering strategies to induce such molecules to adopt acentric structures. Etter points out that a single hydrogen bond is intrinsically non-centrosymmetric so that non-centrosymmetric arrays can be constructed from judiciously designed molecular building blocks joined by strategically positioned hydrogen bonds. In designing materials suitable for second-harmonic generation, the approach is to choose a molecule with features promoting a large value of $\beta$, and to arrange hydrogen-bond donors and acceptors in positions that promote noncentrosymmetric aggregation.

One notable success of this strategy, reported by Etter and Frankenbach earlier this year ${ }^{3}$, is the co-crystal of $p$ aminobenzoic acid and 3,5-dinitrobenzoic acid, which has the packing depicted in the figure. As predicted, the molecules form a non-centrosymmetric, two-dimensional sheet which is held together by an extensive network of hydrogen bonds. The stacking of these acentric, polar layers is harder to predict: the individual layers are, in fact, buckled so that they stack on top of one another with translational symmetry. The polarity of the layer is, therefore, transferred to the crystal as a whole and the material is capable of secondharmonic generation. (Unfortunately, the molecular dipole and a crystallographic 2 -fold axis coincide, resulting in a loss of phase-matchability, and hence of performance.)

Several other approaches for inducing molecules into non-centrosymmetric environments are being explored. For example, thiourea inclusion compounds containing certain organometallic complexes - such as $\left(\eta^{6}\right.$-benzene $) \mathrm{Cr}(\mathrm{CO})_{3}$ - can give pronounced second-harmonic generation ${ }^{4}$. The pure (native) crystalline phases of these organometallic materials are centrosymmetric and therefore incapable of second-harmonic generation, but when included within the linear tunnels of their thiourea inclusion compounds, they adopt a non-centrosymmetric headto-tail dipolar alignment. The inclusion of an organic molecule ( $p$-nitroaniline) within an acentric aluminophosphate molecular sieve (ALPO-5) has also been used to produce second-harmonic generation $^{5}$.

Another important study ${ }^{6}$ has shown that 'guest' molecules occluded within centrosymmetric 'host' crystals can be selectively incorporated into certain symmetry related sectors of the crystal but not into others. Even extremely small amounts ( 0.01 per cent weight) of guest can lead to a sufficient lowering of the symmetry of the crystal for significant second-harmonic generation to be possible.

Kenneth D. M. Harris is in the Department of Chemistry, University of St Andrews, St Andrews KY16 9ST, UK and Mark D. Hollingsworth is in the Department of Chemistry, University of Alberta, Edmonton, Alberta T6G 2G2, Canada.

1. Desiraju, G. R. Crystal engineering: the design of organic solids (Elsevier, Amsterdam, 1989).

. Zyss, J. \& Oudar, J.L. Phys. Rev. A26, 2028-2048 (1982) 3. Etter, M.C. \& Frankenbach, G.M. Chem. Mater. 1, 10-12 (1989).

Tam, W et al Chem. Mater. 1, 128-140 (1989).

5. Cox, S.D., Gier, T.E., Stucky, G.D. \& Bierlein, J. J. Am chem. Soc. 110, 2986-2987 (1988).

6. Weissbuch, I., Lahar, M., Leiserowitz, L., Meredith, G. R. \& Vanherzeele, H. Chem. Mater. 1, 114-118 (1989). 